\title{
Effect of Cardiac Rehabilitation on Blood Pressure in Stable Angina Patients
}

\author{
Mazumdar', Soma, Kumar ${ }^{2}$, Ashok, Verma ${ }^{3}$, S.K. \\ ${ }^{\mathbf{1} E x}$ Prof., Department of Physiotherapy, Baba Farid University, Faridkot, Punjab, India. Email: \\ mazumdars20@yahoo.com \\ ${ }^{2}$ Assistant Professor, Department of Sports Science, Punjabi University Patiala-147001, Punjab, India \\ ${ }^{3}$ Professor, Department of Sports Science, Punjabi University Patiala-147001, Punjab, India
}

\section{Abstract}

The purpose of this study was to investigate the effect on systolic and diastolic blood pressures after six-week cardiac rehabilitation programme in the stable angina patients. A randomly chosen thirty male patients (experimental group) of stable angina age ranged from 40 to 60 years were given a six- weeks cardiac rehabilitation programme and thirty male patients of stable angina (control group) age ranged from 40 to 60 years were not given any cardiac rehabilitation. The systolic and diastolic blood pressure was measured during TMT (before and after) the cardiac rehabilitation. Results showed a statistical significant improvement in the systolic and diastolic blood pressure after cardiac rehabilitation in the experimental group but the control group showed no significant improvement.

Key Words: Stable Angina, Systolic Blood Pressure, Diastolic Blood Pressure, Cardiac Rehabilitation, TMT

\section{Introduction}

In more than $90 \%$ of patients, stable angina is caused by a greater than $70 \%$ obstruction in at least one coronary artery. In less than $10 \%$ of individuals, a lesser degree of atheromatous obstruction, coronary artery spasm or small vessel disease is present. During periods of exercise to exertion, catecholamine release causes an increase in heart rate, an increase in the velocity and force of myocardial contraction producing an elevation in Blood Pressure (BP), and an increase in myocardial oxygen demand (Khan, 2006). In the presence of a significant coronary arterial stenosis, an oxygen deficit occurs. Myocardial ischemia increase catecholamine release, resealing in additional increase in heart rate and blood pressure, with further oxygen lack and the vicious cycle ensues. In addition the coronary arteries fill during the diastolic period, which is shortened during tachycardia. Blood factors, including lipoproteins and platelet functions, and arterial wall functions, with its effect on coagulation ,blood pressure and organ perfusion influence the processes of arteriosclerosis, atherosclerosis and thrombosis, which can had to CAD . Coronary artery disease, along with these other processes may result in angina, MI or death.

\section{Materials and Method}

A randomly sampled 60 patients of stable angina were evaluated for this study. They were divided into two groups- experimental and control, each was comprised of 30 patients. The experimental group was explained aerobic exercises for thirty minutes once a day for four days a week for six weeks. The intensity suggested for the exercises to 
these patients were calculated by Karvonen's formula.

For the first two weeks, THR = RHR $+31 \%$ (HR max - RHR)

for the second two weeks, THR = $\mathrm{RHR}+35 \%$ (HR max - RHR)

and for the last two weeks THR = RHR +40\% (HR max - RHR)
The control group was not given any cardiac rehabilitation programme and was allowed only leisure life style. The systolic and diastolic blood pressure was measured during TMT on the first day. The patients of both the groups had undergone two TMTs (one TMT on the first day and the other after 6 -weeks).

Results and Discussion

Table 1. Mean \pm SD of Blood Pressure at standing \& during TMT (before \& after cardiac rehabilitation) of experimental group

\begin{tabular}{|c|c|c|c|c|c|c|}
\hline Variables & CARDIAC REHAB. & STAGE & & $\mathbf{N}$ & MEAN & SD \\
\hline \multirow{8}{*}{$\begin{array}{l}\text { Systolic } \\
\text { Blood } \\
\text { Pressure }\end{array}$} & \multirow{4}{*}{ BEFORE } & STANDING & & 30 & 135.7 & 9.52 \\
\hline & & & 1 & 30 & 144.73 & 8.38 \\
\hline & & & 2 & 30 & 154.2 & 7.45 \\
\hline & & & 3 & 30 & 163.96 & 6.34 \\
\hline & \multirow{4}{*}{ AFTER } & STANDING & & 30 & 133.2 & 9.12 \\
\hline & & & 1 & 30 & 142.57 & 7.94 \\
\hline & & & 2 & 30 & 150.7 & 7.03 \\
\hline & & & 3 & 30 & 159.38 & 6.9 \\
\hline \multirow{8}{*}{$\begin{array}{l}\text { Diastolic } \\
\text { Blood } \\
\text { Pressure }\end{array}$} & \multirow{4}{*}{ BEFORE } & STANDING & & 30 & 97.53 & 9.61 \\
\hline & & & $\mathbf{1}$ & 30 & 103.2 & 6.74 \\
\hline & & & 2 & 30 & 107.5 & 6.33 \\
\hline & & & 3 & 30 & 112.07 & 5.2 \\
\hline & \multirow{4}{*}{ AFTER } & STANDING & & 30 & 94.87 & 7.96 \\
\hline & & & $\mathbf{1}$ & 30 & 100.3 & 5.58 \\
\hline & & & 2 & 30 & 103.5 & 5.28 \\
\hline & & & 3 & 30 & 107 & 4.3 \\
\hline
\end{tabular}

Table 2. Mean \pm SD of Blood Pressure at standing \& during TMT (before \& after cardiac rehabilitation) of control group

\begin{tabular}{|c|c|c|c|c|c|c|}
\hline VARIABLE & CR & STAGE & & $\mathbf{N}$ & MEAN & SD \\
\hline \multirow{7}{*}{$\begin{array}{l}\text { Systolic } \\
\text { Blood } \\
\text { Pressure }\end{array}$} & \multirow{4}{*}{ BEFORE } & \multicolumn{2}{|l|}{ STANDING } & 30 & 137.63 & 8.8 \\
\hline & & & 1 & 30 & 147.57 & 8.26 \\
\hline & & & 2 & 30 & 158.17 & 8.27 \\
\hline & & & 3 & 30 & 166.47 & 5.7 \\
\hline & \multirow{3}{*}{ AFTER } & STANDING & & 30 & 135.33 & 8.16 \\
\hline & & & 1 & 30 & 146.53 & 7.97 \\
\hline & & & 2 & 30 & 157.67 & 8.87 \\
\hline
\end{tabular}




\begin{tabular}{|c|c|c|c|c|c|c|}
\hline & & & 3 & 30 & 165.93 & 5.69 \\
\hline \multirow{8}{*}{$\begin{array}{l}\text { Diastolic } \\
\text { Blood } \\
\text { Pressure }\end{array}$} & \multirow{4}{*}{ BEFORE } & \multicolumn{2}{|l|}{ STANDING } & 30 & 101.23 & 8.41 \\
\hline & & & 1 & 30 & 106.37 & 5.84 \\
\hline & & & 2 & 30 & 110.2 & 4.09 \\
\hline & & & 3 & 30 & 114.57 & 3.59 \\
\hline & \multirow{4}{*}{ AFTER } & STANDING & & 30 & 99.63 & 8.08 \\
\hline & & & 1 & 30 & 106.4 & 6.02 \\
\hline & & & 2 & 30 & 109 & 4.87 \\
\hline & & & 3 & 30 & 113.9 & 3.6 \\
\hline
\end{tabular}

Table 3. Comparison (paired t-test) of Blood Pressure during TMT (before (B) \& after (A) 6-weeks of cardiac rehabilitation) of experimental \& control groups

\begin{tabular}{|c|c|c|c|c|c|c|c|}
\hline GROUP & Variable & STAGE & $\begin{array}{l}\text { B/A } \\
\text { Before/After }\end{array}$ & $\mathbf{N}$ & $\begin{array}{l}\text { MEAN } \\
\text { DIFF. }\end{array}$ & SD & $\mathbf{t}$ \\
\hline \multirow{8}{*}{ EXPERIMENTAL } & \multirow{4}{*}{$\begin{array}{l}\text { Systolic } \\
\text { Blood }\end{array}$} & STAND & B/A & 30 & 2.5 & 2.05 & 6.69 \\
\hline & & $\mathbf{1}$ & $\mathbf{B} / \mathbf{A}$ & 30 & 2.17 & 2.44 & 4.87 \\
\hline & & 2 & B/A & 30 & 3.5 & 2.6 & 7.38 \\
\hline & & 3 & B/A & 30 & 4.59 & 3.62 & 6.82 \\
\hline & \multirow{4}{*}{$\begin{array}{l}\text { Diastolic } \\
\text { Blood }\end{array}$} & STAND & $\mathbf{B} / \mathbf{A}$ & 30 & 2.67 & 3.69 & 3.96 \\
\hline & & $\mathbf{1}$ & B/A & 30 & 2.9 & 4.63 & 3.43 \\
\hline & & 2 & $\mathbf{B} / \mathbf{A}$ & 30 & 4 & 6.12 & 3.57 \\
\hline & & 3 & B/A & 30 & 5.07 & 4.47 & 6.1 \\
\hline \multirow{8}{*}{ CONTROL } & \multirow{4}{*}{$\begin{array}{l}\text { Systolic } \\
\text { Blood }\end{array}$} & STAND & B/A & 30 & 2.3 & 1.88 & 6.71 \\
\hline & & 1 & $\mathbf{B} / \mathbf{A}$ & 30 & 1.03 & 2.41 & 2.34 \\
\hline & & 2 & $\mathbf{B} / \mathbf{A}$ & 30 & 0.5 & 3.24 & 0.84 \\
\hline & & 3 & B/A & 30 & 0.53 & 3.06 & 0.95 \\
\hline & \multirow{4}{*}{$\begin{array}{l}\text { Diastolic } \\
\text { Blood }\end{array}$} & STAND & B/A & 30 & 1.6 & 2.48 & 3.52 \\
\hline & & 1 & B/A & 30 & 3.33 & 2.93 & 0.06 \\
\hline & & 2 & $\mathbf{B} / \mathbf{A}$ & 30 & 1.2 & 2.48 & 2.34 \\
\hline & & 3 & $\mathbf{B} / \mathbf{A}$ & 30 & 0.67 & 3.05 & 1.19 \\
\hline
\end{tabular}

Table 1 and Table 2 show the mean values of systolic blood pressure and diastolic blood pressure of the experimental group and the control group. It was observed that the systolic blood pressure and the diastolic pressure rise steadily in standing position from stage-1 to stage- 3 of TMT in both the groups. As

Date of Communication: Dec. 7, 2013

Date of Acceptance: Jan. 23, 2014 in both the groups subjects were hypertensive, the systolic blood pressure at stage- 3 of TMT reaches a maximum limit. The range of systolic blood pressure at $120-129 \mathrm{~mm} / \mathrm{Hg}$ is considered to be normal and $130-139 \mathrm{~mm} / \mathrm{Hg}$ is considered high normal (Dwyer and Davis 2005). Thus, the results of this study can be 
described that all the subjects were above normal range irrespective of being under medications. Table 1 and Table 2 shows that there was a steady rise in the diastolic pressure in standing position from stage 1 to stage -3 . A number of studies have shown that a doubling in the cumulative incidence of cardiovascular events in those with a high normal systolic blood pressure $(120-139 \mathrm{~mm} / \mathrm{Hg})$ as compared with those with a normal systolic blood pressure 120mm/Hg (Vasan et al., 2001). The diastolic blood pressure of both the groups was also rising from the stage- 1 to stage-3 of TMT.According to ACSM the normal diastolic blood pressure is $80-84$ $\mathrm{mm} / \mathrm{Hg}$, high normal $85-89 \mathrm{~mm} / \mathrm{Hg}$, stage 1 (Mild) hypertension $90-99 \mathrm{~mm} / \mathrm{Hg}$ and stage 2 (moderate) 100-109 $\mathrm{mm} / \mathrm{Hg}$ (Dwyer and Davis 2005). The increased prevalence of hypertension in metabolic syndrome is the increase in the concentration of free fatty acids that is associated with resistance to the action of insulin in peritoneal adipocyte, worsens inflammation, oxidative stress and endothelial function and increases systolic blood pressure and diastolic blood pressure (Umpierrez et al., 2009). The high range of diastolic blood pressure has been related with the increase in the peripheral vascular resistance due to atherosclerosis but may not be an accurate predictor of cardiovascular risk than systolic blood pressure (Rosendorff, 2006).
Table 3 shows the significant differences in the experimental group of before and after the cardiac rehabilitation during the stages of TMT and observed no significant differences in the control group. The systolic and diastolic blood pressure in standing position showed significant differences in both experimental group and control group. During exercise the sympathetic activation increases with increase in exercise. The sympathetic activation leads to tachycardia and ionotropic effect in the heart. This probably is due to the cardiac rehabilitation exercises administered to the experimental groups while no such rehabilitation given to the control group. In other words, up to stage 3 of this protocol, the blood pressure response seems to be physiologically justified. It may be further justified that the increment in exercise intensity may affect the blood pressure response of the subject.

During exercise there was a sympathetic activation which led to the vasoconstriction of the vessels of the peripheral large muscles. But due to the exercise of the muscles there was a vasodilatation. It is also observed that the subjects due to autonomic dysfunction there was an increase in peripheral resistance. It is the imbalance between these factors that led to increase the diastolic BP with the increase in the stages (1-3) during the TMT. The control group showed the maximum increased in the diastolic BP as these subjects had not 
undergone any cardiac rehabilitation and so had lesser vasodilatation. As the systolic blood pressure is an excellent marker of left ventricular function during exercise and systolic blood pressure is expected to rise during aerobic exercise; either graded (TMT) or steady state, constant load exercise(Dwyer and Davis 2005). The systolic blood pressure rise during exercise provides information about the hemodynamic response to increasing physical stress. Dynamic exercise provokes a large increase in the systolic blood pressure without much change in diastolic blood pressure (McHam et al., 1999). The Systolic blood pressure rise primarily reflects an increase in cardiac output during dynamic exercise. A high rise in systolic blood pressure during exercise may be a consequence of high exercise capacity. The systolic blood pressure may remain elevated for a longer time if sympathetic tone does not decrease and the vagal tone does not increase during post exercise period and may decrease more after exercise in fit and healthy person (Palatini 1998). It has been observed that most of the anti- hypertensive drugs usually lower only the resting systolic blood pressure and the beta-blockers attenuates the magnitude of the rise in systolic blood pressure during dynamic exercise as well as lowers the resting blood pressure (Chick et al., 1988). The diastolic blood pressure is determined mainly by cardiac output and peripheral vascular resistance. During exercise, cardiac output increases and peripheral vascular resistance decreases in response to vasodilatation of resistance vessels within exercising skeletal muscles. An increase in diastolic pressure is therefore may be a result of inappropriate high cardiac output or impaired vasodilatation of resistance vessels within the skeletal muscles. Hypercholesterolemia is strongly associated with impaired reactivity to endothelium dependent and endothelium independent vasodilators. Vasodilatation of resistance vessels in muscle during exercise is influenced by several endothelium independent mediators including nitric oxide, prostaglandins, adenosine and other metabolically linked vasodilators such as potassium and hydrogen ions. Hypercholesterolemia may inhibit the factors that causes vasodilatation and thus may cause an increase in diastolic pressure (Brett et al., 2000).

Conclusion: It was concluded that sixweek cardiac rehabilitation shows a positive effect on the blood pressure i.e. a decrease in the systolic and diastolic blood pressure of the subjects in the experimental group than the control group.

\section{References}

Brett, S.E., Ritter, J.M., Chowienezyk, P.J.. 2000. Diastolic blood pressure changes during exercise positively correlate with serum cholesterol and insulin resistance. Circulation 101: 611-615.

Chick, T.W., Halperin, A.K., Gacek, E.M. 1988. The effect of anti hypertensive medications on exercise performance: A review. Med. Sci. Sports Exerc., 20: 447-454.

Dwyer, G.B., Davis, S.E. 2005. ACSM's Health. Related Physical Fitness Assessment Manual. 
Lippincott Williams \& Wilkins 4: 35-36, 4748, 111-112.

Khan, G.M. 2006. Contemporary Cardiology: Heart disease Diagnosis and Therapy: A Practical Approach. Second edition; Humana press Inc. Totowa, NJ. 136-138, 148.

McHam, S.A., Marwick, T.H., Pashkow, F.J., Lauer, M.S. 1999. Delayed systolic blood pressure recovery after graded exercise: an independent correlate of angiographic coronary disease. $J$ Am. Coll. Cardiol., 34: 754-759.

Palatini, P. 1998. Exaggerated blood pressure response to exercise pathophysiologic mechanism and clinical relevance. $J$ Sports Med Phy Fitness.38: 1-9.

Rosendorff, C. 2006. Essential Cardiology: Hypertension. Humana Press. 595.

Umpierrez, G.E., Smiley, D., Robalino, G. 2009. Intravenous intralipid- induced blood pressure elevation and endothelial dysfunction in obese African- Americans with type-2 diabetes. $J$ Clin Endocrinol Metab. 94: 609-614.

Vasan, R.S., Larson, M.G., Leip, E.P. 2001.Impact of high normal BP on the risk of cardiovascular disease. N. Engl. J. Med., 345: 1291-1297. 